\title{
CORONAVIRUS INFECTION AND DEMYELINATION
}

\section{Sequence Conservation of the S-Gene during Persistent Infection of Lewis-Rats}

\author{
Helmut Wege, ${ }^{1}$ Albert Stühler, ${ }^{2}$ Hans Lassmann, ${ }^{3}$ and Hanna Wege ${ }^{1}$ \\ ${ }^{1}$ Institute of Diagnostic Virology \\ Federal Research Centre for Virus Diseases of Animals \\ Friedrich-Loeffler-Institutes \\ D-17498 Isle of Riems \\ ${ }^{2}$ Ludwig Institute for Cancer Research \\ London W2 1PG, England \\ ${ }^{3}$ Institute of Neurology \\ A-1090 Vienna, Austria
}

\section{ABSTRACT}

Coronaviruses display a large phenotypic variability, which may be an important factor for diversification and selection. Previous studies have demonstrated that the S-protein is an essential determinant of virulence and pathogenicity. Therefore we studied the S-gene as an indicator molecule for selection processes employing two different MHV-JHM variants. First, Lewis-rats were infected with MHV-JHM-Pi, a variant that causes demyelinating disease after several weeks p. i. It was not possible to isolate infectious MHV-JHM-Pi from such rats, although viral proteins were expressed. The S-gene was rescued directly from brain tissue employing RT-PCR technology. The amplicons were sequenced in bulk or at the level of single clones. We detected no evidence for an increase of S-gene mutants during the length of time. Only few mutations were found at the clonal level. The changes were distributed throughout the analysed S-gene fragments without a predilection in their location. The frequency of mutation remained low within a range of 0.03 to 0.5 mutations per thousand nucleotides. As a second approach, we sequenced the S-genes of viruses isolated from brain tissue infected with MHV-JHM-ts43. Infection of adult Lewis rats with that mutant resulted several weeks to months p.i. in demyelinating encephalomyelitis. The S-gene of this virus contains an insertion of $423 \mathrm{bp}$ in the $\mathrm{S} 1$ region, which is identical to a polymorphic region described for MHV-4. In contrast to JHM-Pi, infectious MHV-JHM-ts43 was readily to iso- 
late from brain tissue. The S-gene sequences of virus isolated 45-106 days p.i. from diseased rats were identical with that of the input virus. These results show, that during a persistent infection of Lewis-rats the S-gene was highly conserved.

\section{INTRODUCTION}

The single stranded, positive sense RNA genome of Coronaviridae comprises a size of $31 \mathrm{~kb}$. Transcription and gene expression are mediated by 5-8 subgenomic RNAs, which are produced by a discontinuous RNA synthesis process. Because of the infidelity of the RNA polymerase newly generated viral RNA can display mutations, deletions and changes are introduced by recombination (Rowe et al., 1997a; Kottier et al., 1995; LaMonica et al., 1991; Banner et al., 1990; Parker et al., 1989). The resulting virus population in an infected host may therefore comprise a heterogenous but defined distribution of molecular variants, termed as "quasispecies". Experimental results and field observations led to the hypothesis, that a quasispecies distribution of RNA virus populations may provide the basis for diversification (Smith et al., 1997; Holland, 1992).

Infections of mice and rats with murine coronaviruses provide fascinating experimental systems to study demyelination and persistence in the central nervous system (Wege, 1995; Kyuwa \& Stohlman, 1990). The mouse hepatitis virus MHV-JHM in particular causes in Lewis-rats a subacute demyelinating encephalomyelitis (SDE) characterized by inflammation and selective loss of myelin which resemble the lesions characteristic for multiple sclerosis of humans (Barac-Latas et al., 1997; Wege et al., 1984 and 1983). Coronaviruses are composed of up to five major structural proteins. These are the nucleocapsid protein $(\mathrm{N})$, the membrane protein (M), the small membrane protein (sM), the hemagglutinin esterase protein (HE), and the large surface glycoprotein (S or spike). Results from a number of studies have shown, that both the S- and HE-protein are important for the neuropathogenicity of the virus and for the ability to infect specific cell types (LaMonica et al., 1991; Parker et al., 1989). The S-protein is very important for receptor binding, cell fusion and induction of the immune response. In tissue culture, virus variants had been selected in the presence of neutralizing antibodies which displayed a significant loss of virulence. The S-gene of such escape variants are characterised by mutations and deletions (Gallagher et al., 1990; Wege et al., 1988; Dalziel et al., 1986; Fleming et al., 1986). It is conceivable, that coronavirus induced chronic infection and demyelination is promoted by evasion of humoral and cellular immune responses by antigenic variation. Based on these observations a number of recent studies focused on the S-protein gene as an indicator molecule for selection processes in vivo (Rowe et al., 1997a,b; Stühler et al., 1997; Pewe et al., 1996; Adami et al., 1995). The association between chronic disease and the role of genetic heterogeneity in virus persistency is an interesting but controversial discussed issue. We investigated the S-gene variability during persistent infection in Lewis-rats employing two different MHV-JHM variants and alternative experimental approaches. As an unexpected result we found that under the constraints of a persistent infection the S-gene sequence was highly conserved and no evidence for a quasispecies distribution was found.

\section{MATERIALS AND METHODS}

MHV-JHM-Pi was derived from a persistently infected cell line (Baybutt et al., 1984). MHV-JHM-ts43 is a temperature sensitive mutant selected after mutagenesis with fluoruracil 
(Wege et al., 1984 and 1983). Both variants are closely related to MHV-JHM wildtype (Schmidt et al., 1987). Specific pathogen free Lewis-rats were intracerebrally inoculated, either with MHV-JHM-Pi at an age of 4-5 days or with MHV-JHM-ts43 at an age of 3-5 weeks. For histology, animals were perfused with $4 \%$ paraformaldehyde in $0.1 \mathrm{M}$ phosphate buffer. Parts of brain and spinal cord were embedded in paraffin. To obtain specimens for RTPCR, animals were dissected and left half of the brain frozen in liquid nitrogen before storage at $-80^{\circ} \mathrm{C}$. The right half of the brain and spinal cord was further processed for histology and immunocytochemistry as described previously (Barac-Latas et al., 1997; Wege et al., 1984 and 1983). Processing of tissue for RT-PCR, selection of primer pairs for defined regions of the S-gene, cloning and sequencing was performed according to Stühler et al., 1997).

\section{RESULTS}

\subsection{Neurovirulence of MHV-JHM-Pi and MHV-JHM-ts43 for Lewis-Rats}

The tissue culture adapted MHV-JHM wild-type strain usually causes within 6-12 days p.i. an acute encephalomyelitis, independent of the age of the rat at time of infection (Wege et al., 1983 and 1984). By contrast, the variant MHV-JHM-Pi replicates in the brain of adult Lewis-rats without causing disease (Baybutt et al., 1984). It was also not virulent for adult mice. If 4-5 days old rats were infected, about $25 \%$ of the animals developed subacute demyelinating encephalomyelitis (SDE) within 20 to 50 days p.i., the majority of rats remained healthy (Stühler et al., 1997). The brain tissue of rats with SDE contained high amounts of virus antigen and developed demyelinating lesions in the white matter of the central nervous system (Barac-Latas et al., 1997). It was not possible to isolate infectious virus from brain homogenates of rats with SDE, although it was easy to isolate virus within $6-8$ days p.i. during the acute phase.

The temperature sensitive mutant MHV-JHM-ts43 was used for infection of adult Lewis-rats (Wege et al. 1983 and 1984). These animals developed SDE as late as several weeks to months p.i. and revealed pronounced lesions of primary demyelination. It was most interesting, that a number of rats developed a relapse of SDE after recovery from disease. In contrast to the results with MHV-JHM-Pi, infectious virus was easily isolatable from tissue of brain and spinal cord.

\subsection{Sequencing of the S-Gene of the Variant MHV-JHM-Pi and MHV-JHM-ts43}

In order to detect changes and to examine variability in the S-genes isolated from infected animals we first determined the consensus sequence of MHV-JHM-Pi from three individual plaques of the input virus. Exactely as described for the MHV-JHM wild-type, the S-gene of MHV-JHM-Pi comprises 3705 nucleotides coding for a protein of 1235 amino acids (Schmidt et al., 1987). In comparison to MHV-JHM wild-type, the S-gene of MHV-JHM-Pi contains 14 nucleotide substitutions (point mutations) which all do result in amino acid changes. These changes were distributed throughout the S-protein and not limited to domains. In comparison to the JHM strain MHV-4 (Parker et al., 1989) both the Sgene of MHV-JHM-Pi and the MHV-JHM wild-type have a deletion of 423 nucleotides starting at position 1359 and extending through to nucleotide 1781 . 
The complete S-gene of the MHV-JHM-ts43 used for infection of Lewis rats was sequenced for comparison with the other MHV-strains. The S-gene consensus sequence of MHV-JHM-ts43 appeared to be almost identical with MHV-4. This sequence is $4128 \mathrm{nu}-$ cleotides long. In comparison to MHV-JHM and MHV-JHM-Pi this virus contains 423 additional nucleotides starting at position 1359 and extending through position 1781 of the S-gene (Parker et al., 1989). Position 763, 764 and 765 were found to be GCT instead of CTG in agreement with the sequences published by Schmidt et al. (1987). The position 3990 was changed from $C$ to $T$. This mutation results only in a silent mutation (amino acid $\mathrm{Ala} \rightarrow$ Ala).

\subsection{S-Gene Sequence Variation in Lewis-Rats Persistently Infected with MHV-JHM-Pi}

Rats were infected with MHV-JHM-Pi and brain tissue of animals suffering from subacute demyelinating encephalomyelitis collected for the analysis of the S-gene without any cell culture passage. The RNA was extracted, transcribed to cDNA and the S-gene was amplified by PCR in three overlapping fragments covering the entire gene. In total, brain tissue from six rats was examined for this analysis (Stühler et al., 1997). The amplified S-gene fragments derived fom brain tissue were exactely of the same size than corresponding S-gene amplicons from the input virus propagated in tissue culture. The consensus sequences of the S-genes derived from brain tissue were identical with the sequence of the input virus.

Because individual mutations at the molecular level might not influence the consensus sequence, sequencing studies of cloned PCR products were performed to reveal a possible quasispecies nature of the S-gene. According to results from previous studies, those gene fragments were selected for clonal analysis which should encompass regions involved in virus neutralization and cell fusion. The brain tissue from six rats with SDE was employed for this analysis. From each animal 20 individual molecular clones per each fragment were sequenced. The RT-PCR amplification products were cloned and then sequenced. We detected some mutations at the clonal level, but the total number of nucleotide substitutions was very low (51 mutations in 360 clones). The distribution of the detected mutations gave no indication for "hot spots" or "clustering" in their location. In total, $181.2 \mathrm{~kb}$ were sequenced ( $30.2 \mathrm{~kb}$ per animal and $10 \mathrm{~kb}$ per single fragment). The number of nucleotide substitutions was by statistical analysis smaller than the theoretical error rate of Taq polymerases ( 0.5 mutations per thousand nucleotides). As described in detail by Stühler et al. (1997), even attempts to drive selection by application of a neutralizing antibody in vivo did not influence the rate of mutations. Their frequency remained always within a range between 0.03 to 0.5 mutations per thousand nucleotides.

\subsection{S-Gene Sequence Variability of MHV-JHM-ts43 Isolates from Diseased Lewis-Rats}

As an alternative approach we investigated the S-gene variability of MHV-JHM-ts43 reisolated from persistently infected rats. In contrast to MHV-JHM-Pi, this variant is virulent for adult mice and rats, and infectious virus was readily isolatable from brain tissue. Furthermore, its S-gene contains the same insert of 423 nucleotides than MHV-4, which appears to be polymorphic and hypervariable (Gallagher et al., 1990). We concentrated on five MHV-JHM-ts43 isolates from brain tissue of Lewis-rats, which developed SDE after 
an incubation time between $45,48,54,95$, and 106 days p. i. respectively (Wege et al., 1984 and 1983). The virus stocks propagated for anaylysis of the S-gene were kept within 2-4 cell passages at most. These virus stocks displayed both the temperature sensitive phenotype and neurovirulence as the input virus. The complete S-gene was sequenced in eight overlapping RT-PCR amplicons. In summary, we could not detect any diversity of the consensus sequence of the S-gene of these reisolates in comparison to the MHV-JHMts 43 input virus. Again, these results are implicating, that the S-gene of coronavirus MHVJHM is strongly conserved under the conditions of a persistent infection in vivo. In total, $20.6 \mathrm{~kb}$ of information were sequenced.

\section{DISCUSSION}

It is well documented by a number of studies that at least the S-gene and HE-gene of coronaviruses are very polymorphic and this variability may play an important role for changes in virulence and during pathogenesis (LaMonica et al., 1991; Gallagher et al., 1990; Wege et al., 1988; Dalziel et al., 1986; Fleming et al., 1986). According to the sequence data on variability the S-gene appears to be an interesting indicator molecule to reveal a quasispecies distribution within the "virus swarm" and it may be a major target for selection based on an enhanced mutant frequency. At least in the mouse system, good evidence had been obtained for an association of genetic variability and persistency in vivo (Rowe et al., 1997a,b; Pewe et al., 1996; Adami et al., 1995). However, some controversy remains in the interpretation of the biological meaning of the observed variability.

The observed genetic stability of the MHV-JHM-Pi S-gene in vivo may reflect a bottleneck situation valid as an exception for this persistent infection. First, MHV-JHM-Pi was only infectious for newborn rats. The immune system of this animals was not fully developed at the timepoint of infection and although virus antigen was detectable in the brain tissue of diseased rats, it was not possible to isolate infectious virus. It is conceivable, that the lack of proper receptors on the host cells of adult rats allows only the survival of the already well adapted S-protein of MHV-JHM-Pi fitting to the rat variant of the MHV-receptor. Second, coronavirus persistence requires a continuous synthesis of viral proteins and the virus may spread slowly within the infected organs. Under these conditions the number of replication cycles may be relatively small and a high mutation frequency of the multifunctional S-protein may disrupt this balanced virus-host relationship. Third, MHV-JHM-Pi is derived from the MHV-JHM wild-type strain which contains a Sgene differing from the JHM strain MHV-4 by a deletion of 423 nucleotides (Rowe et al., 1997b; Parker et al., 1989; Schmidt et al., 1987). It had been shown recently, that S-protein deletion mutants accumulate during persistent infection of mice and that this "hypervariable region" is involved in forming a secondary stem-loop structure, which may be a "hot spot" for RNA recombination (Rowe et al., 1997a,b). We were interested to analyse the S-gene variability of MHV-JHM-ts43 because it contains this 423 nucleotide insert as described for MHV-4 (Parker et al., 1989). Furthermore, in contrast to MHV-JHM-Pi this variant can infect adult rats with a mature immune system and infectious virus could be isolated from diseased rats. However, at least at the level of the consensus sequence, no Sgene changes between the input virus and the isolated virus following a long period of persistency was found.

It has been shown by a number of studies that neutralizing antibodies can drive selection of escape variants, which display an attenuated virulence (Wege et al., 1988; Dalziel et al., 1986; Fleming et al., 1986). In MHV-4 the "hypervariable region" mentioned 
above behaved highly polymorphic in tissue culture experiments under the selection pressure by neutralizing antibodies (Gallagher et al., 1990). Following antibody selection, the S-gene of escape variants displayed deletions in this region. In our case, even by mimicking an immunological selection pressure with a neutralizing antibody in vivo no increase in the frequency of mutations was found (Stühler et al., 1997). On the other hand, depending on the host genetic context the cellular immune response may be a much stronger factor driving selection during chronic disease. In a mouse model for MHV-JHM induced demyelination, mutations were defined especially in the region coding for an immunodominant epitope recognized by cytotoxic T-cells (Pewe et al., 1996).

Taken together, our results appear to be controversial in view of the genetic heterogeneity described in the studies employing mice. On the other hand, only about $50 \%$ of the mice harbored S-gene mutants with deletions, but all animals were persistently infected (Rowe et al., 1997b). Therefore, the impact of genetic heterogeneity of an individual virus population for the outcome of disease and the emergence of variants may differ with the particular virus-host combination. Our results illustrate that a genetically quite heterogenuous virus may remain in an evolutianary silent state during persistence in vivo. RNA replication may be reduced while persisting in infected animals in contrast to acute infections or during replication in tissue culture. These findings are compatible with a possibly high rate and frequency S-gene mutations during natural spread by acute infections in large host populations. The background of neutral mutations may be an unpredictible factor allowing evolution and emergence of new variants involving recombination, deletions and selection by host factors (Baric et al., 1997; Kottier et al., 1995; LaMonica et al., 1991; Gallagher et al., 1990). The study of virus-host relations during persistency and the implications for health and disease remains a fascinating topic.

\section{ACKNOWLEDGMENTS}

The work was supported by the Deutsche Forschungsgemeinschaft and Hertie Stiftung.

\section{REFERENCES}

Adami, C., Pooly, J., Glomb, J., Stecker, E., Fazal, F., Fleming, J.O. and Baker, S.C., 1995, Evolution of mouse hepatitis virus (MHV) during chronic infection: Quasispecies nature of the persisting MHV RNA, Virologi 209: 337-346.

Banner, L.R., Keck, G.K. and Lai, M.M.C., 1990, A clustering of RNA recombination sites adjacent to a hypervariable region of the peplomer gene of murine coronavirus, Virol. 175: 548-555.

Barac-Latas, V., Suchanek, G., Breitschopf, H., Stühler, A., Wege, H. and Lassmann, H., 1997, Patterns of oligodendrocyte pathology in coronavirus induced subacute demyelinating encephalomyelitis in the Lewis rat, Glia 19: 1-12.

Baric, R., Yount, B., Hensley, L., Peel, S.A. and Chen, W., 1997, Episodic evolution mediates interspecies transfer of a murine coronavirus, J. Virol. 71: 1946-1955.

Baybutt, H.N., Wege, H., Carter, M.J. and ter Meulen, V., 1984, Adaptation of coronavirus JHM to persistent infection of murine Sac(-) cells, J. Gen. Virol. 65: 915-924.

Dalziel, R.G., Lampert, P.W., Talbot, P.J. and Buchmeier, M.J., 1986, Site-specific alterations of murine hepatitis virus type 4 peplomer glycoprotein E2 results in reduced neurovirulence, J. Virol. 59: 463-471.

Fleming, J.O., Trousdale, M.D., El-Zaatari, F.A.K., Stohlman, S.A. and Weiner, L.P., 1986, Pathogenicity of antigenic variants of murine coronavirus JHM selected with monoclonal antibodies, J. Virol. 58: 869-875.

Gallagher, T.M., Parker, S.E. and Buchmeier, M.J., 1990, Neutralization-resistant variants of a neurotropic coronavirus are generated by deletions within the aminoterminal half of the spike glycoprotein, J. Virol. 64: 731-741. 
Holland, J.J. (editor, 1992). Genetic diversity of RNA viruses. Curr. Top. Microbiol. Immunol. Springer-Verlag, Berlin-Heidelberg-New York 176.

Kyuwa, S. and Stohlman, S.A., 1990, Pathogenesis of a neurotropic murine coronavirus, strain JHM in the central nervous system of mice, Semin. Virol. 1: 273-280.

Kottier, S., Cavanagh, D. and Britton, P., 1995, Experimental evidence of recombination in coronavirus infectious bronchitis virus, Virologi 213: 569-580.

Lamonica, N., Banner, L.R., Morris, V.L. and Lai, M.M.C., 1991, Localization of extensive deletions in the structural genes of two neurotropic variants of murine coronavirus JHM, Virol. 182: 883-888.

Parker, S.E., Gallagher, T.M. and Buchmeier, M.J., 1989, Sequence analysis reveals extensive polymorphism and evidence of deletions within the E2 glycoprotein gene of several strains of murine hepatitis virus, Virologi 173: $664-673$

Pewe, L., Wu, F.G., Barnett, E.M., Castro, R. and Perlman, S., 1996, Cytotoxic T cell-resistant variants are selected in a virus-induced demyelinating disease, Immunity 5: 253-262.

Rowe, C.L., Fleming, J.O., Nathan, M.J., Sgro, J.-Y., Palmenberg, A. and Baker, S.C., 1997a, Generation of coronavirus spike deletion variants by high-frequency recombination at regions of predicted RNA secondary structure, J. Virol. 71: 6183-6190.

Rowe, C.L., Baker, S.C., Nathan, M.J. and Fleming, J.O., 1997b, Evolution of mouse hepatitis virus: Detection and characterization of spike deletion variants during persistent infection, J. Virol. 71: 2959 -2969.

Schmidt, I., Skinner, M.A. and Siddell, S.G., 1987, Nucleotide sequence of the gene encoding the surface projection glycoprotein of the coronavirus MHV-JHM, J. Gen. Virol. 68: 47-56.

Smith, D.B., McAllister, J., Casino, C. and Simmonds, P., 1997, Virus "quasispecies": making a mountain out of a molehill? J. Gen. Virol. 78: 1511-1519.

Stühler, A., Flory, E., Wege, H., Lassmann, H. and Wege, H., 1997, No evidence for quasispecies populations during persistence of the coronavirus mouse hepatitis virus JHM: sequence conservation within the surface glycoprotein gene S in Lewis rats, J. Gen. Virol. 78: 747-756.

Wege, H., 1995, Immunopathological aspects of coronavirus infections, Springer Semin. Immunopathol. 17: 133-148.

Wege, H., Watanabe, R. and ter Meulen, V., 1984, Relapsing subacute demyelinating encephalomyelitis in rats in the course of coronavirus JHM infection, J. Neuroimmunol. 6: 325-336.

Wege, H., Koga, M., Watanabe, R., Nagashima, K. and ter Meulen, V., 1983, Neurovirulence of murine coronavirus JHM temperature sensitive mutants in rats, Infect. Immun. 39: 1316-1324.

Wege, H., Winter, J. and Meyermann, R., 1988, The peplomer protein E2 of coronavirus JHM as a determinant of neurovirulence: definition of critical epitopes by variant analysis, J. Gen. Virol. 69: 87-98. 